\title{
Société suisse de pédiatrie (SSP)
}

\section{Stephan Rupp}

Co-président de la SSP

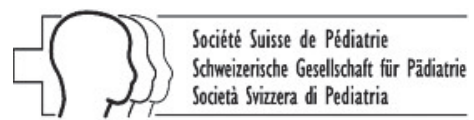

Schweizerische Gesellschaft für Pädiatrie Societa snizera di Pediatria

1 Gauthier E, Tönz O. Esquiss de l'histoire de la Société Suisse de Pédiatrie. Paediatrica. 2001;12(Suppl 6):11-7.

Correspondance:

Dr Stephan Rupp Spitalstrasse 30

CH-8840 Einsiedeln

Tél. 0554121777

stephan_rupp@bluewin.ch
La pédiatrie est devenue une discipline médicale autonome au cours du XIX ${ }^{\mathrm{e}}$ siècle. En 1867, les premiers cours magistraux en pédiatrie sont donnés à Bâle. S'ensuit l'ouverture d'hôpitaux spécialisés dans les soins aux nourrissons et à la petite enfance et, peu après, l'ouverture des premiers cabinets de pédiatrie.

\section{Date de création}

En 1900, le professeur Combe fait parvenir un questionnaire à ses confrères intéressés. Parmi les 33 personnes qui lui répondent, 23 approuvent la création d'une société de pédiatrie, 5 doutent de son bien-fondé et 5 la refusent. Le $1^{\text {er }}$ juin 1901, la Société suisse de pédiatrie [1] est fondée à Olten. Ensuite, des séances sont organisées chaque année tout d'abord les dimanches et ensuite les samedis. Comme le veut la tradition suisse, le principe d'alternance entre la Suisse alémanique et la Suisse Romande est respecté.

\section{Approche interdisciplinaire}

Il est intéressant de noter qu'au début, les spécialistes en chirurgie pédiatrique et en orthopédie faisaient également partie de la société. Alors que les contacts étaient principalement maintenus dans un premier temps par l'organisation de congrès commun, le réel rapprochement n'a eu lieu que ces dernières années. La création de la foederatio Paedo-medicorum helveticorum (fPmh) en 2006 visait à rapprocher les disciplines médicales pédiatriques. Les membres de ces groupements, des pédiatres, mais aussi des chirurgiens pédiatriques et des pédopsychiatres, ont obtenu un statut d'observateurs à la Chambre médicale.

\section{Titres de formation approfondie et conséquences}

Il est évident que la société a connu des turbulences durant ses 108 années d'existence. L'on se souvient encore des discussions quant à la création de titres de spécialiste en pédiatrie ou des formations approfondies. Contrairement à l'avis de nombreux pédiatres, la Chambre médicale s'est décidée à créer des formations approfondies, ce qui s'est avéré positif pour la pédiatrie. L'identité des pédiatres s'est trouvée renforcée par cette décision, étant donné que les formations approfondies se fondent sur le titre de spécialiste en pédiatrie.

\section{Une société consacrée à la pédiatrie hospitalière et en pratique privée}

Au fil des années, le nombre de pédiatres en pratique privée a augmenté par rapport à celui des pédiatres exerçant à l'hôpital, ce qui a entraîné des luttes pour le pouvoir. En 1988, lors de l'Assemblée générale qui se tint à Zoug, il a été décidé que quatre membres parmi les neuf du comité devaient représenter les pédiatres en pratique privée. Malgré cette mesure, les pédiatres praticiens ne se sentaient toujours pas suffisamment représentés, ce qui les incita à créer en 1995 le Forum de pédiatrie pratique (FPP), composé principalement de pédiatres Suisses alémaniques. Même si les contacts furent difficiles au début, ils sont désormais empreints de collégialité et de respect. Le Forum n'a cependant pas réussi à s'imposer en Suisse romande, probablement en raison de la différence de mentalités et de la séparation moins nette entre la pratique privée et le domaine hospitalier.

Au début et pour un certain temps, la structure de la société prévoyait deux piliers, l'un hospitalier et l'autre pratique. Cette séparation a finalement été levée en 2004, au moment de la révision des statuts. Nous voulons une seule pédiatrie pour toute la Suisse. Et dans ce contexte, nous nous demandons à chaque fois si nous nous définissons comme des médecins de premier recours ou des spécialistes en pédiatrie. Dans un certains sens, nous sommes les deux, ce qui a une incidence sur le service de garde et d'urgence qui s'organise différemment suivant les régions.

\section{Les enfants ont besoin d'un lobby}

La SSP sera confrontée à de nouveaux défis. En Suisse, la politique de la santé est de plus en plus axée sur la personne âgée. C'est pourquoi il importe de créer un lobby visant à défendre l'enfant tant sur le plan de sa santé que de la maladie. Pour les pédiatres, la prévention joue donc un rôle prépondérant. Nous projetons également d'étendre les examens préventifs aux adolescents et d'instaurer un examen de contrôle dès trois ans pour évaluer le développement du langage chez l'enfant. La tâche principale consistera à faire en sorte que chaque enfant puisse consulter un pédiatre, que ce soit à l'hôpital ou en cabinet privé, qu'il soit généraliste ou spécialiste. Un autre défi à relever concernera la collaboration avec les autres médecins de premier recours, qui a lieu actuellement au sein du Collège de médecine de premier recours (CMPR), mais qui sera intensifiée avec la création de l'Union des médecins de premiers recours (HAeCH) où la pédiatrie devra, une fois encore, trouver sa place. 